\title{
Cascade-Type Excitation Energy Relay in Organic Thin-Film Solar
}

\section{Cells}

Musubu Ichikawa $^{1,2, *}$, Daiki Takekawa ${ }^{1}$, Hyeon-Gu Jeon ${ }^{1, \dagger}$, Gilles D. R. Banoukepa ${ }^{1,3}$

${ }^{1}$ Interdisciplinary Graduate School of Science and Technology, Shinshu University, 3-15-1 Tokida, Ueda City, Nagano 386-8567, Japan

${ }^{2}$ Presto, Japan Science and Technology Agency (JST), 4-8-1 Honcho, Kawaguchi, Saitama 332-0012, Japan

${ }^{3}$ Japan Society for the Promotion of Science, 8 ichibancho, Chiyoda, Tokyo 102-8472, Japan

(Received )

We investigated small-molecule-based organic photovoltaic (PV) cells with three different electron-donating material layers, two thiophene/phenylene co-oligomers [ $\alpha, \omega$-bis(biphenyl-4-yl)terthiophene (BP3T) and $\alpha, \omega$-diphenyl sexithiophene (P6T)] and copper phthalocyanine $(\mathrm{CuPc})$, and one electron-accepting material layer $\left(\mathrm{C}_{60}\right)$. A cascade-type energy relay between the three donor layers occurred, and the incident photon-current conversion efficiency improved in the blue light region, where $\mathrm{CuPc}$ has very low optical absorption. An increase in P6T photoluminescence intensity in a 
two-layer sample (BP3T/P6T) on quartz confirmed interlayer excitation transfer (ET) from BP3T and P6T. The bulk heterojunction architecture in our interlayer-ET-based organic PV cell was effective. Moreover, P6T appeared to have a relatively long exciton diffusion length of several tens of nanometers.

Keywords: organic photovoltaics, organic solar cells, phthalocyanine, excitation transfer, thiophene, exciton diffusion length

*Corresponding author at: Interdisciplinary Graduate School of Science and Technology, Shinshu University, 3-15-1 Tokida, Ueda City, Nagano 386-8567, Japan Tel: +81-268-21-5498; Fax: +81-268-21-5417

Email address: musubu@shinshu-u.ac.jp

†Present address: Department of Materials Science and Biotechnology, Graduate School of Science and Engineering, Ehime University, Matsuyama 790-8577, Japan 


\section{Introduction}

In recent years, organic photovoltaic (PV) cells have emerged as a promising solar cell technology; the potential to fabricate flexible, lightweight devices with low material consumption and tunable absorption characteristics allows a cost-efficient electricity supply $[1,2]$. Increasing research efforts have steadily improved the power conversion efficiencies (PCEs) of small-molecule-based organic [3-6] and polymer-based [6-10] PV cells since Tang reported the first efficient bilayer PV device [11]. Because structurally well-defined small-molecule materials avoid the inherent batch-to-batch variations in the physical properties of polymers, they provide better reproducibility. In addition, these materials can be adapted for using vacuum evaporation deposition techniques, which allow well-defined structures to be built layer by layer. Thus, organic small-molecule PV cells fabricated by vacuum evaporation deposition could provide various future improvements to the PCEs of organic PV cells-improvements that still need to make PV cells truly useful.

To enhance the PCE, researchers must first increase the intensity of light absorption and broaden the available spectrum, which could be achieved by using more than two materials with complementary optical absorption properties. Organic PV cells fundamentally require two different materials, an electron-donating and an electron-accepting material, for efficient charge separation. $\mathrm{C}_{60}$ fullerene derivatives, which are generally used as acceptor materials, have weak optical absorption in the visible and near infrared (IR) region owing to their high symmetry. Thus, a donor material used in an organic PV cell should absorb a wider spectral range, from blue to near IR, to achieve a higher PCE. From the viewpoint of the electronic structures of molecules and molecular solids, the electronic levels in organic semiconductors are 
essentially not very broad, and so the optical absorption bands are smaller than those of inorganic semiconductors. In short, organic semiconductors should be colorful materials such as pigments and dyes.

It was recently demonstrated that adding another donor organic semiconductor layer to a conventional organic $\mathrm{PV}$ cell composed of phthalocyanine $(\mathrm{Pc})$ and $\mathrm{C}_{60}$ fullerene (with a planar heterojunction) could enhance its incident photon-current conversion efficiency (IPCE) around the blue light region in which Pc has no optical absorption [12]. Two thiophene derivatives, $\alpha, \omega$-bis(biphenyl-4-yl)terthiophene (BP3T) and $\alpha, \omega$-diphenyl sexithiophene (P6T) (Fig. 1), were used as additional donor materials. Both absorb strongly in the blue light region, i.e., their optical absorption properties are complementary to those of Pc. In the literature, we confirmed that interlayer excitation transfer $(\mathrm{ET})$ from BP3T or P6T to copper Pc $(\mathrm{CuPc})$ occurred, and this interlayer ET relays the photonic energy absorbed by the additional thiophene layer to the $\mathrm{CuPc}$ layer, resulting in IPCE sensitization. In short, using two-layer donor stacks has the potential to improve organic PV performance.

Indeed, several groups have reported layered organic PVs composed of two donor layers [13-19]. Although different mechanisms, such as double exciton dissociation at the heterojunction of a centered ambipolar donor layer with both the acceptor layer and the other donor layer, have been proposed [13, 14, 18], an interlayer ET sensitization mechanism has been accepted $[15,16,19]$. For example, Hong et al. demonstrated interlayer ET of triplet excitons between pentacene and zinc Pc [15], and Reusswig et al. reported interlayer ET-sensitized singlet fission of rubrene [19]. Schlenker et al. recently extended this double donor layer stack system to a three-layered one composed of tetracene and its phenyl-substituted derivatives; the 
primary effect was to increase the open-circuit voltage $\left(V_{\mathrm{OC}}\right)$ by reducing recombination of holes and electrons [20]. However, in the three-layered donor stack, a donor layer placed farthest from the acceptor layer, corresponding to the donor layer on the left in Fig. 1a, works only as an exciton-blocking layer; furthermore, photon-to-electron conversion due to optical absorption and a subsequent cascade-type energy relay (Fig. 1a) has not been confirmed. Hence, in this study we investigated a cascade-type energy relay between three donor layers on the basis of interlayer ET to investigate the possibility of further improvements in the intensity and/or spectral width of the IPCE. In addition, we examined the effectiveness of the bulk heterojunction (BHJ) architecture in our interlayer-ET-based energy-relay-type organic PV cells. We also investigated the exciton diffusion length of P6T.

\section{Experimental}

Figure $1 \mathrm{~b}$ shows a schematic of the device structure used in the study and Fig. 1c shows the chemical structures of the materials used. The cells are composed of three different donor material layers. One of the donor layers is in direct contact with an acceptor layer. These two layers are indispensable for charge separation; we used CuPc and $\mathrm{C}_{60}$ as donor and acceptor materials, respectively. We employed BP3T and P6T as the materials for the two additional donor layers, which provide light absorption but not charge separation. Thiophene/phenylene co-oligomers such as BP3T and P6T reportedly possess the good hole-transport characteristics necessary for the additional donor layers [21-24]. BP3T and P6T (Sumitomo Seika Industries), $\mathrm{C}_{60}$ (Frontier Carbon), and BCP (TCI) were purified by temperature-gradient train sublimation with an Ar gas flow before use. We used CuPc (organic light-emitting device grade) donated by Nippon 
Steel Chemicals without further purification.

We prepared the devices by depositing several materials sequentially onto commercially available indium-tin-oxide (ITO)-coated glass substrates having a sheet resistance of $15 \Omega /$ square. The substrates were first washed with aqueous detergent, pure water, and 2-propanol with ultrasonification, and then treated with $\mathrm{O}_{2}$ plasma at 50 W for 5 min. We prepared a poly(3,4-ethylenedioxythiophene) poly(styrenesulfonate) (PEDOT:PSS) layer on the substrates by spin-coating a commercially available formula (H.C. Starck, Clevios P AI 4083) before depositing several organic layers by thermal evaporation under vacuum $\left(<2.0 \times 10^{-4} \mathrm{~Pa}\right)$ at a rate of $0.5 \AA / \mathrm{s}$. Finally, an aluminum electrode was prepared on top of the organic layer stack by thermal evaporation in vacuum at a rate of $10 \AA / \mathrm{s}$. The active areas of the cells were approximately $6 \mathrm{~mm}^{2}$. We transferred the fabricated devices into a highly inert glove box $\left(\mathrm{O}_{2}\right.$ concentration: $5 \mathrm{ppm}$, dew point: $<-50^{\circ} \mathrm{C}$ ) with no exposure to ambient conditions and then mounted them in a small chamber with a quartz window in the glove box.

A source meter (Keithley, 2410) measured the devices' current density versus voltage $(J-V)$ curves with $100-\mathrm{mW} / \mathrm{cm}^{2}$ artificial global solar light irradiation at air mass 1.5 global (AM1.5G) from a Newport Oriel solar simulator (Model 91160) with an AM1.5G filter (Newport Oriel, 81088) and a light intensity stabilizer (Newport Oriel, Model 68945). The power density of the irradiation was measured with a pyroelectric optical power meter (Ophir, P3). We recorded the IPCE spectra of the devices with a source meter (Keithley 2400) under monochromatic light irradiation from a light source (Bunko Keiki SM-25A). The intensity of the monochromatic light at each wavelength was measured with a calibrated silicon photodiode (Hamamatsu Photonics S1337-1010BQ). Absorption and photoluminescence (PL) spectra of the thin-film 
samples were recorded with a Shimadzu spectrophotometer (UV-9150) and a Systems Engineering PL quantum efficiency measurement system (QEMS-2000), respectively.

Sample films were deposited by thermal evaporation on quartz substrates. Surface topological and phase images of the films were recorded with a Bruker Dimension Icon PTX atomic force microscope (AFM) in tapping mode. Note that in this study, we employed relatively thin layers of $\mathrm{CuPc}$ and $\mathrm{C}_{60}$, compared to those in other studies of organic PVs with $\mathrm{CuPc}$ and/or $\mathrm{C}_{60}$, because our goal was to determine exactly how the interlayer excitation energy transfer influences organic PV devices.

\section{Results and Discussion}

Figure 2 shows the IPCE spectra of cells with BP3T thicknesses $(Y)$ of $0,5,10$, 20, and $30 \mathrm{~nm}$ and a fixed 10-nm P6T thickness $(X)$, and of a reference cell without either a BP3T or P6T layer. Most noticeable from the figure is that the reference and the cell with only a 10-nm-thick P6T layer exhibited an increased IPCE across almost the entire wavelength region. We have already reported the source of the improvement as follows: (1) spectral sensitization at short wavelengths because of interlayer ET from P6T to $\mathrm{CuPc}$ and (2) buffer effects at long wavelengths [12]. When BP3T layers were introduced, the IPCE near 350-500 nm changed, although the IPCE owing to CuPc (near $600 \mathrm{~nm}$ ) remained largely unchanged. In short, IPCE in the shorter wavelength region increased as the BP3T layer thickness increased from 5 to $20 \mathrm{~nm}$. BP3T absorbs blue light but not red light (Fig. 3). Thus, the increase in the blue light IPCE is probably due to optical absorption by the BP3T layer, which was placed between the PEDOT:PSS and P6T layers.

To confirm the contribution of the BP3T layer to the IPCE spectra shown in 
Fig. 2, we subtracted the IPCE spectrum of the cell with a 10-nm-thick BP3T layer from that of the cell with a 20 -nm-thick one. In the resulting difference spectrum, a distinctive shoulder appeared near $500 \mathrm{~nm}$, which is also observed in the absorption spectrum of BP3T. The BP3T layer did not directly touch the $\mathrm{C}_{60}$ acceptor layer; in fact, there were two other layers $\left(\mathrm{P} 6 \mathrm{~T}\right.$ and $\mathrm{CuPc}$ ) between the $\mathrm{BP} 3 \mathrm{~T}$ and $\mathrm{C}_{60}$ layers. Thus, direct conversion of the BP3T excitons to charge carriers because of charge transfer to $\mathrm{C}_{60}$ could not occur. Therefore, excitation energy transfer from the BP3T layer to the $\mathrm{CuPc}$ layer may be the cause of charge separation.

It seems impossible that the BP3T exciton traveled to the $\mathrm{CuPc}$ directly because of the thickness of the P6T interlayer $(10 \mathrm{~nm})$, which is much larger than the conventional Förster radii (a few nanometers) of good excitation energy donor and acceptor combinations [25]. In fact, the estimated Förster radius $\left(R_{0}\right)$ of BP3T and CuPc was $4.1 \mathrm{~nm}$ according to the following equation [26]:

$$
R_{0}^{6}=\frac{9 \eta_{P L} \kappa^{2} \ln 10}{128 \pi^{5} n^{4} N_{A}} \int f_{D}(\lambda) \varepsilon_{A}(\lambda) \lambda^{4} d \lambda
$$

where $\eta_{\mathrm{PL}}, \kappa, n, N_{\mathrm{A}}, f_{\mathrm{D}}, \varepsilon_{\mathrm{A}}$, and $\lambda$ are the PL quantum yield of BP3T without $\mathrm{CuPc}$, the orientation factor, the refractive index of the media, Avogadro's constant, the normalized PL spectrum of BP3T, the molar extinction coefficient spectrum of CuPc [27], and the wavelength, respectively. The $\eta_{\mathrm{PL}}$ value of BP3T thin films was experimentally found to be $5 \%$, and $\kappa^{2}$ and $n$ were assumed to be $2 / 3$ and 2 , respectively.

Although $R_{0}$ for BP3T and CuPc is sufficiently smaller than the thickness of the P6T layer, the ET rate constant also depends on the excitation energy acceptor conditions (for example, isolated molecules in an inert medium, a two-dimensional 
molecular sheet [monolayer], or a three-dimensional molecular layer [slab]) $[28,29]$. The case reported here is considered a slab, and the ET rate constant $\left(k_{\mathrm{F}}\right)$ can be expressed as a function of the distance $(x)$ from the excitation energy donor molecule to the front surface of the acceptor slab as follows [25]:

$$
k_{F}(x)=\frac{C_{A}}{\tau} \frac{\pi}{6} \frac{R_{0}^{6}}{x^{3}}
$$

where $C_{\mathrm{A}}$ is the energy acceptor molecular density [19] and $\tau$ is the exciton lifetime of the donor. A $k_{\mathrm{F}}$ value of $4.3 / \tau$ was obtained when $x$ was $10 \mathrm{~nm}$ (corresponding to the thickness of the P6T layer). This value shows that the excitation energy transfer process from the BP3T layer to the CuPc layer was not negligible even when the 10-nm-thick P6T layer was placed between them. However, $R_{0}$ for BP3T and P6T was estimated to be $3.3 \mathrm{~nm}$ [27], and the P6T layer was adjacent to the BP3T layer. Consequently, excitation energy transfer from BP3T to P6T should be dominant in our situation. Note that for transfer from BP3T to a P6T slab at a distance $x$ of $1 \mathrm{~nm}, k_{\mathrm{F}}$ was calculated to be 935/ $\tau$. In addition, $R_{0}$ for $\mathrm{P} 6 \mathrm{~T}$ and $\mathrm{CuPc}$ was $3.4 \mathrm{~nm}$, where $\eta_{\mathrm{PL}}$ for $\mathrm{P} 6 \mathrm{~T}$ was experimentally determined to be $1 \%$. The relatively large $R_{0}$ for P6T and CuPc and direct contact between the layers ensures efficient ET between the two.

Figure 4 shows the PL spectra of two different BP3T and P6T layer samples. In one sample, 30-nm-thick P6T and BP3T layers were deposited sequentially on a quartz substrate (BP3T/P6T/quartz), and in the other, 30-nm-thick BP3T was deposited on a quartz substrate placed on a 30-nm-thick P6T layer on another quartz substrate (BP3T/quartz/P6T/quartz), as shown in the figure inset. We employed these two samples to compensate for the reabsorption effect, which should become marked in the equipment used in this study because it has an integrating sphere. The excitation light $(\lambda$ 
$=378 \mathrm{~nm})$ from an ultraviolet LED $(378 \mathrm{~nm})$ was incident from the BP3T side, but it excited both BP3T and P6T because of the thinness of the BP3T layer. Therefore, both BP3T and P6T contributed to the PL spectra of both samples. However, the shapes of the spectra differed. The BP3T/P6T/quartz sample showed stronger PL, especially near 600-700 nm, and weaker PL at $525 \mathrm{~nm}$, the first apparent shoulder. Because the PL of P6T appears at wavelengths longer than that of BP3T, which is also shown in the figure, we concluded that the BP3T/P6T/quartz emitted stronger P6T PL than the other sample. Each PL spectrum could be adequately reproduced as a weighted summation of the PL spectra of single BP3T and P6T layers, and the results showed that for the BP3T/P6T/quartz sample, P6T contributed about three times more to the spectrum than for the BP3T/quartz/P6T/quartz sample. Only the BP3T/P6T/quartz sample demonstrated the potential for ET from BP3T to P6T because BP3T directly touches the P6T layer in that sample. Consequently, interlayer ET from BP3T to P6T and sequential cascade interlayer ET from BP3T to CuPc via P6T appear to have occurred in the PV cells (Fig. 3).

Figure 5 shows the $J-V$ characteristics of the cells whose spectra are shown in Fig. 2. The short-circuit current density $\left(J_{\mathrm{SC}}\right)$ increased with increasing BP3T thickness when the thickness was less than $20 \mathrm{~nm}$, and then $J_{\mathrm{SC}}$ decreased. The change in $J_{\mathrm{SC}}$ was clearly consistent with the change in the IPCE spectra shown in Fig. 2. On the other hand, the open-circuit voltage $\left(V_{\mathrm{OC}}\right)$ also changed as the BP3T thickness increased. No $V_{\mathrm{OC}}$ change was expected, and in fact, no $V_{\mathrm{OC}}$ change was observed in our earlier interlayer-ET-sensitized organic PV [12]. The averaged $V_{\mathrm{OC}}$ values of several cells fabricated in this study are plotted in the inset of Fig. 5. The averaged values ranged around $0.36-0.39 \mathrm{~V}$ and seemed to depend somewhat on the BP3T thickness. However, 
the origin of the dependence is currently unclear and hence further investigations are required.

Next, the $J-V$ curves of the cells with a thick BP3T layer, e.g., $20 \mathrm{~nm}$, reveal that the fill factors (FFs) of the cells decrease compared with that of the cell with no BP3T. The inset also shows the BP3T thickness dependence of the averaged FF values of several cells. The FFs of the cells with a BP3T layer, except for the 5-nm-thick BP3T cell, clearly exhibited a continuous decrease. The BP3T-thickness-dependent decrease in the FF implies that the hole mobility of BP3T is not very high. A previous study on the hole mobility of BP3T single crystals revealed that BP3T has relatively high hole mobility $\left(0.29 \mathrm{~cm}^{2} / \mathrm{Vs}\right)[30]$ in the $a b$ plane of its monoclinic crystal structure $(a: 7.5262$ $\AA, b: 5.7856 \AA, c: 59.997 \AA, \beta: 92.818^{\circ}$ ) [31]. Although the hole mobility along the $c$-axis has not been reported, it must be smaller than that in the $a b$ plane [32]. It is generally known that straight aromatic molecules such as pentacene, oligothiophene, and oligophenylene form deposited crystalline thin films in which molecules stand upright (c-axis orientation) [33-36]. A similar thin-film orientation of BP3T probably caused the relatively low hole mobility along its thickness direction and caused the BP3T-thickness-dependent decrease in the FF. The lack of an FF decrease in the 5-nm-thick BP3T cell will be discussed later in the paper.

Figure 6 shows AFM images of the thin films prepared on the same ITO substrate with a PEDOT:PSS layer. As shown in panel a, numerous BP3T grains, with sizes varying from tens of nanometers to a hundred nanometers, appeared and covered nearly all of the underlying PEDOT:PSS surface. Note that the BP3T thickness dependence of the FF shown in Fig. 5 inset indicates that the 10-nm-thick BP3T film can cover nearly the entire underlying surface. This is because the $200-\mathrm{meV}$-high 
influential hole injection barrier at the BP3T/P6T interface (Fig. 1c) was not effective in the 5-nm-thick BP3T cell but was effective in the 10-nm-thick one. According to our previous study, a barrier with a height of less than $100 \mathrm{meV}$ had little effect on charge transport at the interface, but the influence became larger when the height exceeded 100 meV [12]. Panel b shows the surface morphology of the P6T layer on the BP3T layer; grains were also observed in the P6T but seemed to be larger than the BP3T layer grains. Although it is difficult to estimate the consistency of the thin (10-nm-thick) P6T layer in this case, we believe that the thicker P6T layers used in this study might cover the surface of the underlying BP3T layers because of the molecular similarity between P6T and BP3T. On the other hand, it is generally known that CuPc can form relatively smooth flat thin films $[37,38]$. Although the topography of the top CuPc layer on the P6T/BP3T bilayer (panel c) was still granular owing to the topography of the underlying surface, the phase image (panel d) of that surface indicated that only a few parts of the underlying P6T layer were not covered by $\mathrm{CuPc}$ (dark areas indicated by white arrows in panel d). This is because phase images taken by tapping mode AFMs reflect the softness and/or adsorptive characteristics of surfaces [39, 40] and are also influenced by variations in surface morphology.

Note that the rather rough granular films shown in Fig. 6 would raise concerns about the coherence of the discussions in this study. However, a surface line profile (Fig. S1) of Fig. 6a, for example, estimates lateral and vertical dimensions of the surface undulation in the BP3T film to be $55 \pm 12 \mathrm{~nm}$ and $3.2 \mathrm{~nm}$ as $R_{\mathrm{a}}$, respectively. The lateral dimension, which can be assumed to represent the average size of the grains, was much larger than the $R_{0}$ values (the Förster radii). In addition, the $R_{\mathrm{a}}$ value was comparable to the $R_{0}$ values. These results indicate that the discussions in this study are not invalidated 
by this concern. However, another concern about direct contact between BP3T and $\mathrm{C}_{60}$ seems to still remain. This is because a few tall grains of BP3T can be seen in Fig. 6 a (for example, three grains on the bottom are almost 30-nm-high), and such tall grains are hardly covered completely with the layers (P6T and $\mathrm{CuPc})$ placed on it. Thus, some part of the tall grains would be in direct contact with $\mathrm{C}_{60}$, and an alternative charge generation pathway via the direct contact seems to be the case. However, short exciton diffusion lengths (from a few to several tens of nanometers) [41, 42] of organic semiconductors can limit areas in which excitons access the interface of $\mathrm{BP} 3 \mathrm{~T} / \mathrm{C}_{60}$ and contribute to charge generation resulting in photocurrent. In contrast, the ET pathway for charge generation can add an additional elongation due to ET to the exciton diffusion length. This means that the ET pathway is somewhat preferable compared with the alternative pathway in the marginal area around the direct contact of BP3T/C 60 . Thus, although it appears that there are a few tall grains resulting in direct contact of $\mathrm{BP} 3 \mathrm{~T}$ and $\mathrm{C}_{60}$ in the cells, the ET pathway for charge generation is dominant. In addition, the area covered by the tall grains in Fig. $6 \mathrm{a}$ is fractional to the view field.

It is generally known that the BHJ architecture, in which donor and acceptor materials are mixed, is useful for overcoming the short exciton diffusion lengths of organic semiconductors and for enhancing the conversion efficiency of the exciton to the charge carrier. We think that the BHJ architecture should be effective in our interlayer-ET-based PV cell structure. The reason is that interlayer ET produces an exciton that is localized near the interface between the ET-energy donor and acceptor (P6T and $\mathrm{CuPc}$ in this study) because the Förster lengths are approximately several nanometers. Figure 7 shows the IPCE spectra of the interlayer-ET-based PV cells with a BHJ architecture. The figure inset illustrates the cell structure. The IPCE increased 
significantly compared with those shown in Fig. 2, indicating that the BHJ is effective in our ET-based PV cells. In addition, the dependence of the IPCE on the BP3T thickness was similar to that of the planar heterojunction cells shown in Fig. 2. The IPCE initially increased with BP3T thickness, especially in the blue light region, but then decreased. Furthermore, the apparent shoulder at $500 \mathrm{~nm}$, which was due to BP3T (as discussed earlier), was observed, especially with thicker BP3T. We can safely conclude that cascade-type interlayer ET from BP3T to CuPc via P6T occurs.

If the thickness of the P6T layer increases beyond its exciton diffusion length, the cascade-type interlayer ET from BP3T to CuPc disappears. Therefore, we varied the thickness of the P6T layer to investigate this behavior. Figure 8 shows the IPCE spectra of cells with the structures shown in Fig. 7 (inset), where $Y$ (the BP3T layer thickness) was fixed at 50 and $X$ (the P6T layer thickness) varied from 20 to 70 . When the P6T layer was less than $60 \mathrm{~nm}$ thick, the IPCE in the blue light region, which is contributed by BP3T, seemed to decrease slightly but was relatively constant. However, when the thickness of the P6T layer increased to $70 \mathrm{~nm}$, the IPCE at a small peak at $540 \mathrm{~nm}$ actually increased (the peak is assigned to P6T and can be observed in Fig. 3). A thick organic semiconductor generally affects the charge extraction process of the electrode because of its rather low carrier mobility, but the increase in IPCE observed at $540 \mathrm{~nm}$ because of P6T indicates that charge extraction should not decrease the IPCE in the blue region owing to $\mathrm{BP} 3 \mathrm{~T}$. The $\mathrm{BP} 3 \mathrm{~T}$ exciton is transferred to the P6T layer, and the P6T exciton, originating from the ET, travels within the P6T layer to the CuPc layer. The P6T layer might disrupt exciton travel when it is thicker than its diffusion length, resulting in decreased IPCE from BP3T. This finding again indicates that the BP3T exciton is transferred to the CuPc layer via the P6T layer, and the thickness of $70 \mathrm{~nm}$ 
might mean that the P6T exciton diffusion length is relatively large compared with that of other organic thin-film semiconductors, which generally have exciton diffusion lengths of several nanometers to a few tens of nanometers [25, 41, 43-45]. More detailed studies need to be conducted to estimate the exciton diffusion length, which requires measurement of the ET interaction lengths between BP3T and P6T and between P6T and CuPc.

\section{Conclusion}

We investigated a cascade-type energy relay between three donor layers composed of two oligothiophenes (BP3T and P6T) and $\mathrm{CuPc}$ on the basis of interlayer ET. A cascade-type energy relay appeared in the system and improved the IPCE value in the blue light region, in which $\mathrm{CuPc}$ has very poor optical absorption. Interlayer ET from BP3T and P6T was examined by comparing the PL spectra of two thin-film samples: BP3T/P6T/quartz and BP3T/quartz/P6T/quartz. In addition, we investigated and confirmed the effectiveness of the BHJ architecture in our interlayer-ET-based organic PV. P6T appears to have a relatively long exciton diffusion length of several tens of nanometers.

Acknowledgments: This work was supported by the JST PRESTO program (Photoenergy Conversion Systems and Materials for the Next Generation Solar Cells).

\section{References}

[1] S. R. Forrest, Nature 428 (2004) 911.

[2] F. C. Krebs, T. Tromholt, M. Jorgensen, Nanoscale 2 (2010) 873. 
Leo, M. Riede, M. Weil, O. Tsaryova, A. Weiß, C. Uhrich, M. Pfeiffer, P. Bäuerle, Adv. Funct. Mater. 21 (2011) 897.

[4] T. Kaji, M. Zhang, S. Nakao, K. Iketaki, K. Yokoyama, C. W. Tang, M. Hiramoto, Adv. Mater. 23 (2011) 3320.

[5] X. Xiao, G. Wei, S. Wang, J. D. Zimmerman, C. K. Renshaw, M. E. Thompson, S. R. Forrest, Adv. Mater. 24 (2012) 1956.

[6] M. A. Green, K. Emery, Y. Hishikawa, W. Warta, E. D. Dunlop, Progress in Photovoltaics: Research and Applications 20 (2012) 12.

[7] C. J. Brabec, S. Gowrisanker, J. J. M. Halls, D. Laird, S. Jia, S. P. Williams, Adv. Mater. 22 (2010) 3839.

[8] Y. Liang, Z. Xu, J. Xia, S.-T. Tsai, Y. Wu, G. Li, C. Ray, L. Yu, Adv. Mater. 22 (2010) E135.

[9] H. Zhou, L. Yang, A. C. Stuart, S. C. Price, S. Liu, W. You, Angew. Chem. 123 (2011) 3051 .

[10] L. Dou, J. You, J. Yang, C.-C. Chen, Y. He, S. Murase, T. Moriarty, K. Emery, G. Li, Y. Yang, Nat. Photon. 6 (2012) 180.

[11] C. W. Tang, Appl. Phys. Lett. 48 (1986) 183.

[12] M. Ichikawa, E. Suto, H.-G. Jeon, Y. Taniguchi, Org. Electron. 11 (2010) 700.

[13] S. Sista, Y. Yao, Y. Yang, M. L. Tang, Z. Bao, Appl. Phys. Lett. 91 (2007) 223508.

[14] G. Zhang, W. Li, B. Chu, L. Chen, F. Yan, J. Zhu, Y. Chen, C. S. Lee, Appl. Phys. Lett. 94 (2009) 143302.

[15] Z. R. Hong, R. Lessmann, B. Maennig, Q. Huang, K. Harada, M. Riede, K. 
Leo, J. Appl. Phys. 106 (2009) 064511.

[16] D. C. Coffey, A. J. Ferguson, N. Kopidakis, G. Rumbles, ACS Nano 4 (2010) 5437.

[17] T. D. Heidel, D. Hochbaum, J. M. Sussman, V. Singh, M. E. Bahlke, I. Hiromi, J. Lee, M. A. Baldo, J. Appl. Phys. 109 (2011) 104502.

[18] K. Cnops, B. P. Rand, D. Cheyns, P. Heremans, Appl. Phys. Lett. 101 (2012) 143301.

[19] P. D. Reusswig, D. N. Congreve, N. J. Thompson, M. A. Baldo, Appl. Phys. Lett. 101 (2012) 113304.

[20] C. W. Schlenker, V. S. Barlier, S. W. Chin, M. T. Whited, R. E. McAnally, S. R. Forrest, M. E. Thompson, Chem. Mater. 23 (2011) 4132.

[21] M. Ichikawa, H. Yanagi, Y. Shimizu, S. Hotta, N. Suganuma, T. Koyama, Y. Taniguchi, Adv. Mater. 14 (2002) 1272.

[22] H. Yanagi, Y. Araki, T. Ohara, S. Hotta, M. Ichikawa, Y. Taniguchi, Adv. Funct. Mater. 13 (2003) 767.

[23] K. Nakamura, M. Ichikawa, R. Fushiki, T. Kamikawa, M. Inoue, T. Koyama, Y. Taniguchi, Jpn J. Appl. Phys. 43 (2004) L100.

[24] S. Hotta, T. Katagiri, T. Yamao, K. Shimizu, H. Yanagi, M. Ichikawa, Y. Taniguchi, Int. J. Polym. Mater. 57 (2008) 515.

[25] S. R. Scully, M. D. McGehee, J. Appl. Phys. 100 (2006) 034907.

[26] T. Förster, Discuss. Faraday Soc. 27 (1954) 7.

[27] To calculate $\varepsilon_{\mathrm{A}}$ and $C_{\mathrm{A}}$, densities of thin films of $\mathrm{CuPc}$ and P6T were assumed to be the same as that of each crystal; $1.643 \mathrm{~g} / \mathrm{cm}^{3}(\mathrm{CuPc})$ and $1.485 \mathrm{~g} / \mathrm{cm}^{3}(\mathrm{P} 6 \mathrm{~T})$.

[28] H. Kuhn, J. Chem. Phys. 53 (1970) 101. 
[30] K. Nakamura, M. Ichikawa, R. Fushiki, T. Kamikawa, M. Inoue, T. Koyama, Y. Taniguchi, Jpn. J. Appl. Phys. 44 (2005) L1367.

[31] S. Hotta, M. Goto, R. Azumi, M. Inoue, M. Ichikawa, Y. Taniguchi, Chem. Mater. 16 (2004) 237.

[32] W.-Q. Deng, W. A. Goddard, J. Phys. Chem. B 108 (2004) 8614.

[33] L. Torsi, A. Dodabalapur, L. J. Rothberg, A. W. P. Fung, H. E. Katz, Science $272(1996) 1462$.

[34] D. J. Gundlach, Y. Y. Lin, T. N. Jackson, D. G. Schlom, Appl. Phys. Lett. 71 (1997) 3853.

[35] I. P. M. Bouchoms, W. A. Schoonveld, J. Vrijmoeth, T. M. Klapwijk, Synth. Met. 104 (1999) 175.

[36] K. Takimiya, Y. Kunugi, Y. Konda, N. Niihara, T. Otsubo, J Am Chem Soc 126 (2004) 5084 .

[37] O. Berger, W. J. Fischer, B. Adolphi, S. Tierbach, V. Melev, J. Schreiber, Journal of Materials Science: Materials in Electronics 11 (2000) 331.

[38] L. Grządziel, J. Żak, J. Szuber, Thin Solid Films 436 (2003) 70.

[39] S. N. Magonov, V. Elings, M. H. Whangbo, Surf Sci 375 (1997) L385.

[40] A. Noy, C. H. Sanders, D. V. Vezenov, S. S. Wong, C. M. Lieber, Langmuir 14 (1998) 1508.

[41] P. Peumans, A. Yakimov, S. R. Forrest, J. Appl. Phys. 93 (2003) 3693.

[42] M. Ichikawa, Thin Solid Films (in press), doi: 10.1016/j.tsf.2012.12.036.

[43] Y. Terao, H. Sasabe, C. Adachi, Appl. Phys. Lett. 90 (2007) 103515.

[44] S. Banerjee, A. P. Parhi, S. S. K. Iyer, S. Kumar, Appl. Phys. Lett. 94 (2009) 
223303.

[45] J. Meiss, M. Hermenau, W. Tress, C. Schuenemann, F. Selzer, M. Hummert, J. Alex, G. Lackner, K. Leo, M. Riede, Phys. Rev. B 83 (2011) 165305.

\section{Figure Captions}

Figure 1. (a) Conceptual energy diagram of PV cell proposed in this study, (b) sectional cell structure, and (c) structures and abbreviations of chemicals used in this study. Values in parentheses indicate the highest occupied molecular orbital levels.

Figure 2. IPCE spectra of cells with different BP3T thicknesses (between 0 and $30 \mathrm{~nm}$ ) and constant P6T thickness $(10 \mathrm{~nm})$. The reference cell had no BP3T or P6T layer. The inset shows a difference IPCE spectrum obtained by subtracting the spectrum of the 10-nm-thick BP3T cell from that of the 20 -nm-thick cell.

Figure 3. Absorption coefficient spectra for thin films of each material. The absorption coefficient was defined as the ratio of the absorbance of each thin film and its thickness.

Figure 4. PL spectra of two different samples composed of BP3T and P6T layers, and the PL spectra of single layers of BP3T and P6T (dashed lines). The intensities of the single layer spectra were normalized to be 4 at each maximum.

Figure 5. Current density-voltage characteristics of the cells having the IPCE spectra shown in Fig. 2. 
Figure 6. Surface topological images (a, b, c) and phase image (d) of thin film samples: (a) BP3T (10 nm), (b) P6T (10 nm, top)/BP3T (10 nm, bottom), and (c, d) CuPc (10 nm, top)/P6T (10 nm, middle)/BP3T (10 nm, bottom). All samples were deposited by thermal evaporation on the same ITO-coated glass substrate with a 30-nm PEDOT:PSS layer. All the images are $500 \mathrm{~nm}^{2}$ in size, and their upper limits (ULs) were (a) $30 \mathrm{~nm}$, (b) $30 \mathrm{~nm}$, (c) $40 \mathrm{~nm}$, and (d) $30^{\circ}$.

Figure 7. IPCE spectra of PV cells with the structure shown in the inset, where $X$ was fixed at 10 and $Y$ was varied from 0 to 50 .

Figure 8. IPCE spectra of PV cells with the structure shown in Fig. 7 (inset), where $X$ was varied from 20 to 70 and $Y$ was fixed at 50 . 

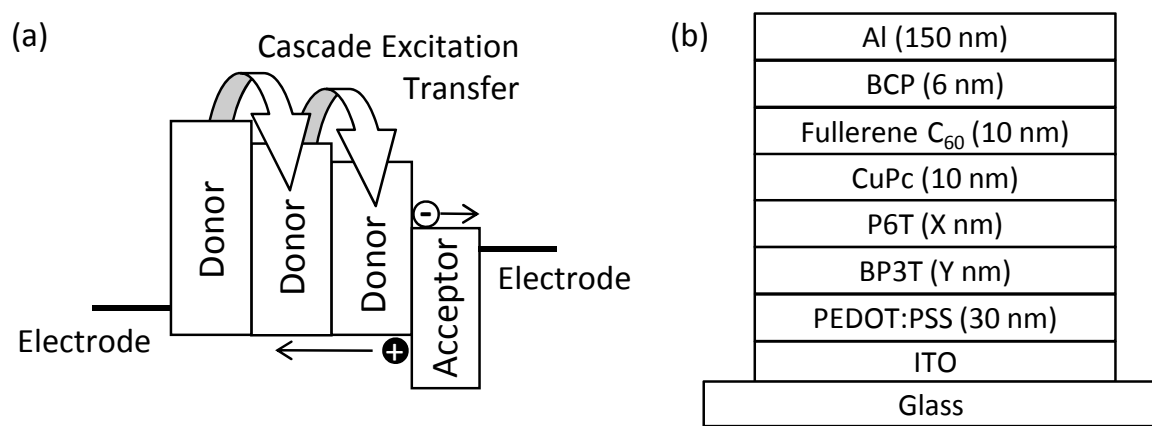

(c)
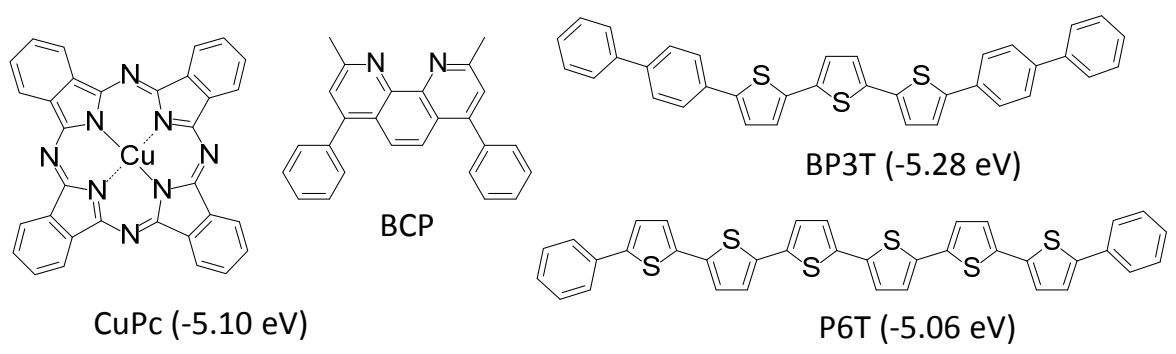

Figure 1. (a) Conceptual energy diagram of PV cell proposed in this study, (b) sectional cell structure, and (c) structures and abbreviations of chemicals used in this study. Values in parentheses indicate the highest occupied molecular orbital levels.

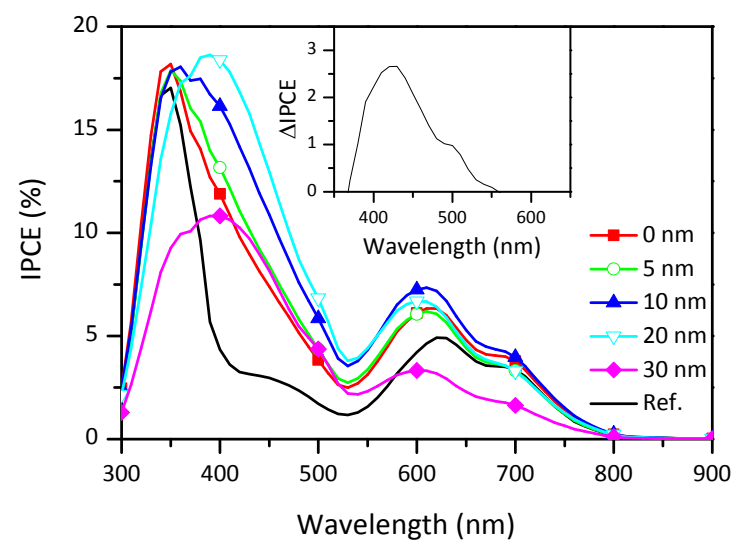

Figure 2. IPCE spectra of cells with different BP3T thicknesses (between 0 and $30 \mathrm{~nm}$ ) and constant P6T thickness $(10 \mathrm{~nm})$. The reference cell had no BP3T or P6T layer. The inset shows a difference IPCE spectrum obtained by subtracting the spectrum of the 10-nm-thick BP3T cell from that of the 20-nm-thick cell. 


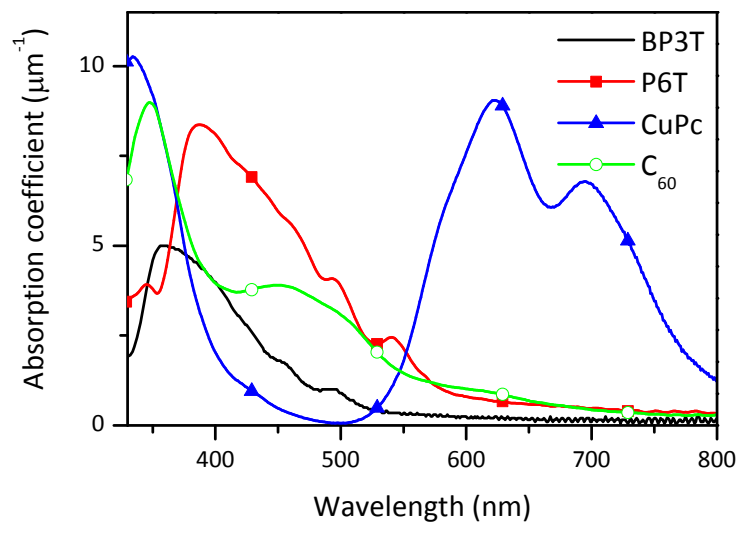

Figure 3. Absorption coefficient spectra for thin films of each material. The absorption coefficient was defined as the ratio of the absorbance of each thin film and its thickness.

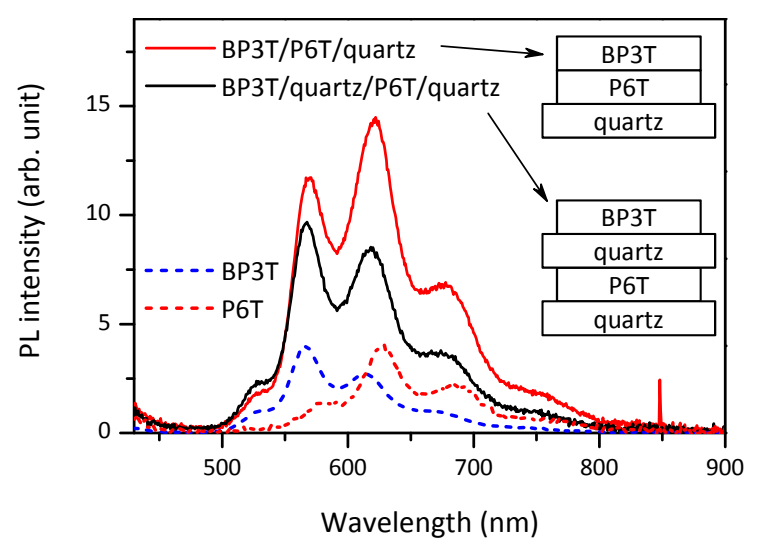

Figure 4. PL spectra of two different samples composed of BP3T and P6T layers, and the PL spectra of single layers of BP3T and P6T (dashed lines). The intensities of the single layer spectra were normalized to be 4 at each maximum. 


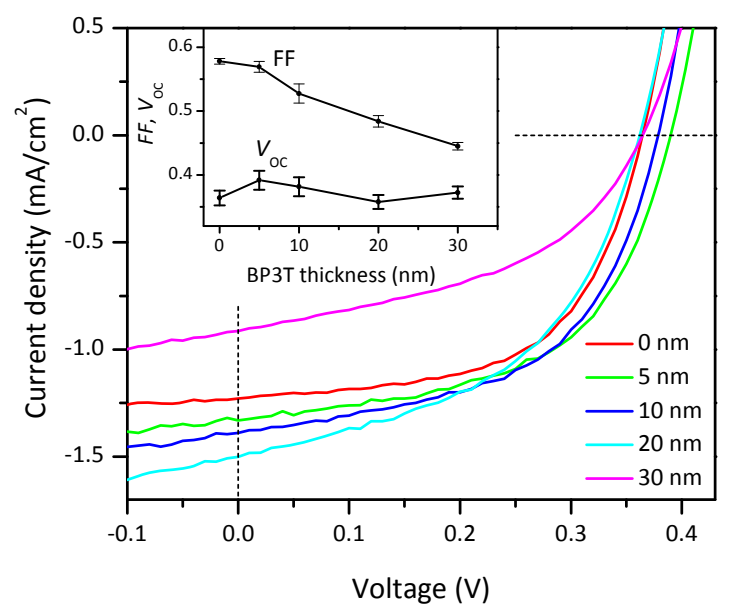

Figure 5. Current density-voltage characteristics of the cells having the IPCE spectra shown in Fig. 2.
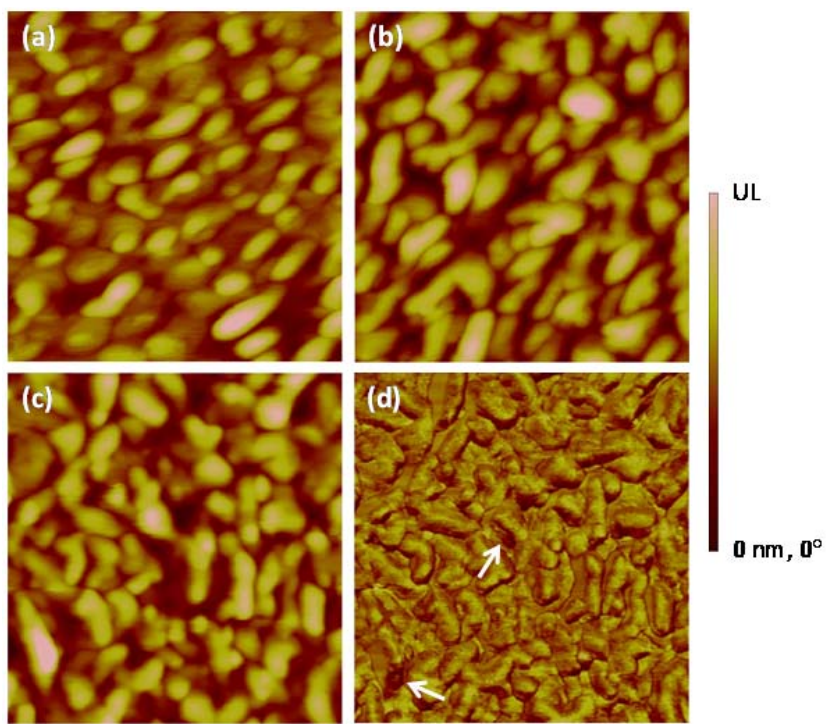

Figure 6. Surface topological images (a, b, c) and phase image (d) of thin film samples: (a) BP3T (10 nm), (b) P6T (10 nm, top)/BP3T (10 nm, bottom), and (c, d) CuPc (10 nm, top)/P6T (10 nm, middle)/BP3T (10 nm, bottom). All samples were deposited by thermal evaporation on the same ITO-coated glass substrate with a 30-nm PEDOT:PSS layer. All the images are $500 \mathrm{~nm}^{2}$ in size, and their upper limits (ULs) were (a) $30 \mathrm{~nm}$, (b) $30 \mathrm{~nm}$, (c) $40 \mathrm{~nm}$, and (d) $30^{\circ}$. 


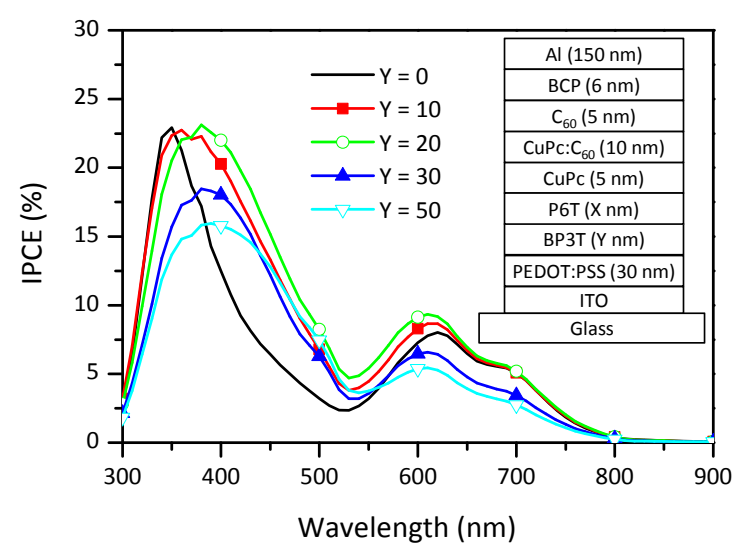

Figure 7. IPCE spectra of PV cells with the structure shown in the inset, where $X$ was fixed at 10 and $Y$ was varied from 0 to 50 .

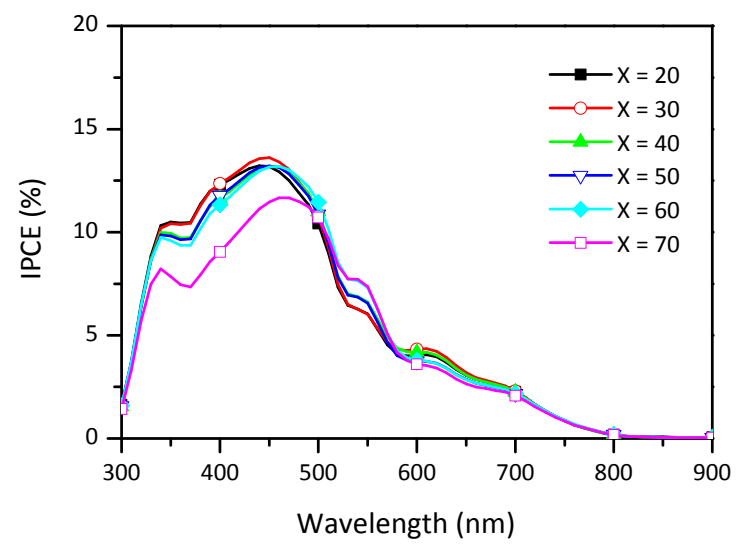

Figure 8. IPCE spectra of PV cells with the structure shown in Fig. 7 (inset), where $X$ was varied from 20 to 70 and $Y$ was fixed at 50 . 


\section{Supplementary Information}

\section{Cascade-Type Excitation Energy Relay in Organic Thin-Film Solar}

\section{Cells}

Musubu Ichikawa, Daiki Takekawa, Hyeon-Gu Jeon, Gilles D. R. Banoukepa

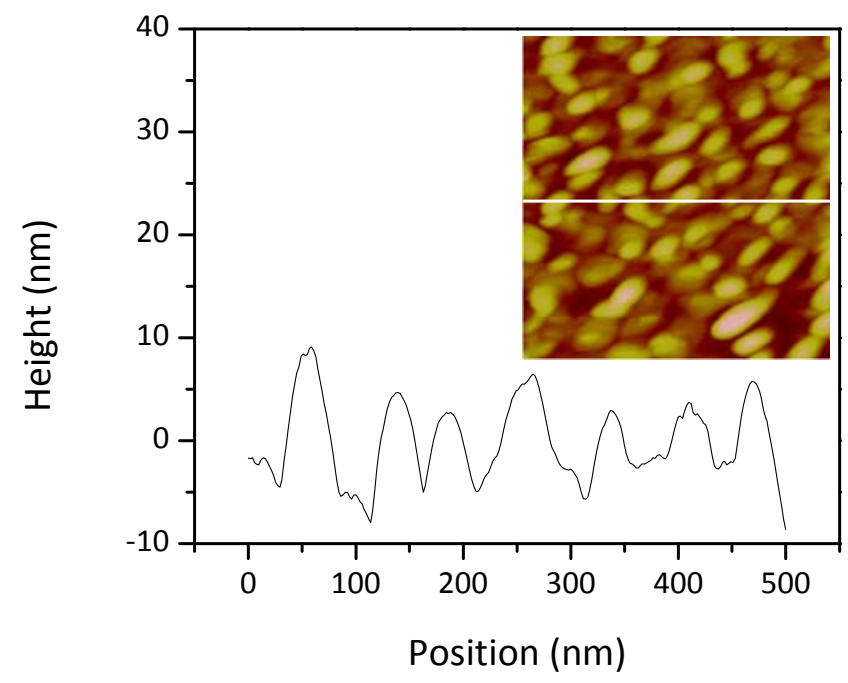

Figure S1. Line profile of the surface of the BP3T film shown in Fig. 6a. Profile was obtained at position indicated by white line in the inset. 